\title{
Aspectos de interés en el diseño de sobredentaduras sobre implantes
}

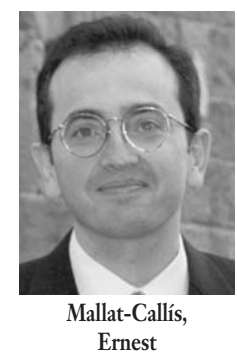

\author{
Interesting details in implant overdentures design
}

\section{Mallat-Calliss, Ernest}

Médico-Odontólogo
Resumen: El diseño de una sobredentadura sobre implantes presenta una serie de aspectos que es conveniente tener presente ya que de ellos depende la supervivencia de la propia prótesis. La biomecánica marca el comportamiento de la sobredentadura cuando está sometida a función y, por tanto, será un factor que determinará el número de implantes a colocar, el tipo de anclaje a utilizar y las características del mismo así como el propio montaje de dientes. Además, la elección del tipo de anclaje irá estrechamente ligada a la determinación de ferulizar o no los implantes y del correcto diseño de las barras se derivará una menor frecuencia de complicaciones en las mismas. Por último, la reabsorción progresiva de la cresta ósea debajo de las sobredentaduras implantomucosoportadas favorece que éstas se apoyen sobre los pilares y es la zona de la prótesis que está ocupada por el sistema de pilares y anclajes la que presenta un menor grosor de acrílico y, por tanto, una baja resistencia. Con el fin de evitar posibles fracturas de la sobredentadura incluiremos siempre un armazón metálico en ella. En este artículo se abordarán todos estos aspectos en detalle.

Palabras clave: Sobredentadura sobre implantes, Biomecánica, Anclajes axiales, Barras, Cantilevers.

Abstract: There are some details in implant overdentures design that can influence their long-term survival. Biomechanics determine the way overdenture works under function, so it will be a main factor to decide the number of implants, type of attachment and teeth mounting procedures. Besides, attachment selection is very closey related to the need of splinting implants and the incidence of complications will depend mainly on the bar design. We also know that, as bone resorption takes place below an implant-mucosa supported overdenture, the load directed to the implants increases, and this area of the overdenture is so thin that it can fracture. For this reason, it is necessary to reinforce the overdenture with a Co-Cr structure. In this article, all this factors will be dealt with in detail.

Key words: Implant overdentures, Biomechanics, Axial attachments, Bars, Cantilevers.

BIBLID [1138-123X (2006)11:3; mayo-junio 265-380]

Mallat-Callís E. Aspectos de interés en el diseño de sobredentaduras sobre implantes. RCOE 2006;11(3):329-343. 


\section{Introducción}

Cuando afrontamos el tratamiento de un paciente mediante una sobredentadura sobre implantes son varios los aspectos protésicos que pueden condicionar el éxito. Un punto en común a todos ellos es la biomecánica de la prótesis, es decir, según se trate de una prótesis implantosoportada o de una prótesis implantomucosoportada, ya que dictará el comportamiento de la sobredentadura cuando sea sometida a la función. La biomecánica determinará el número de implantes a colocar, el tipo de anclaje $y$, en parte, el montaje de los dientes. Además, será conveniente incluir en la prótesis una estructura colada con el fin de reforzarla. Por último, habrá situaciones en las que, a pesar de poderse realizar una prótesis fija implantosoportada será preferible optar por una sobredentadura.

El objetivo de este artículo clínico es analizar cuántos implantes es conveniente colocar, cuándo será preferible una sobredentadura frente a una prótesis fija y qué factores condicionarán la decisión, qué anclaje utilizaremos, por qué colocaremos una estructura de refuerzo en la sobredentadura y qué aspectos del montaje de dientes son importantes.

\section{Nümero de implantes}

En primer lugar, es conveniente decidir cuántos implantes se colocarán ya que influirá en el diseño de la sobredentadura. El número de implantes vendrá determinado tanto por la biomecánica de la prótesis como por la ubicación de la misma,

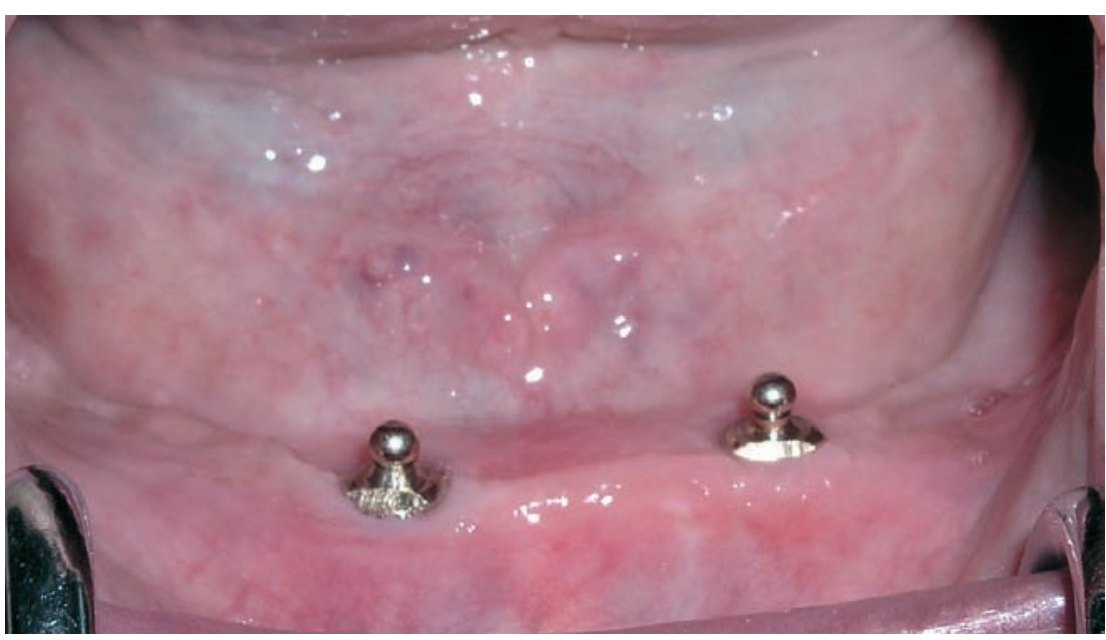

Figura 1. Paciente que presenta una edentación mandibular y al que se la han colocado dos implantes en la zona de los caninos para una sobredentadura sobre anclajes axiales.

en la mandíbula o en el maxilar superior.

En la mandíbula, cuando el objetivo sea una prótesis implantomucosoportada, es suficiente con colocar dos implantes, idealmente situados en la zona de los caninos (fig.1). En su momento se propugnaba la colocación de tres implantes por si acaso se perdía alguno, pero la tasa de éxito en lo que a osteointegración se refiere en la zona mandibular anterior es muy elevada (>96\%) por lo que no está justificado poner uno más por si alguno fallase. Además, un tercer implante nos dificultará en gran medida la colocación de la barra paralela al eje de rotación mandibular cuando este tipo de anclaje sea el elegido. Por último, no se ha demostrado que el hecho de pasar de dos a cuatro implantes determine mejoras en la eficacia masticatoria, ni en la salud periimplantaria ni en las tensiones transmitidas al hueso. Es más, en cuanto a esto último, lo realmente determinante es el diseño y el ajuste de la prótesis.
Si se trata de una prótesis implantosoportada serán necesarios al menos cuatro implantes bien distribuidos. Sólo si han quedado situados muy anteriormente y la extensión anteroposterior de la barra es poca se comportará biomecánicamente como una prótesis implantomucosoportada (fig. 2). Si en cambio los situamos más separados, podremos considerar que el soporte será básicamente implantario.

Todo lo dicho es válido cuando la altura de hueso es suficiente y los implantes colocados tienen más de 10 $\mathrm{mm}$. Si los implantes son de $10 \mathrm{~mm} 0$ menos será preferible colocar cuatro implantes aún cuando la prótesis sea implantomucosoportada. Cuando se trata de implantes tipo Endopore (Innova), implantes aptos para ser colocados en zonas con menor cantidad de hueso, estudios a 10 años certifican que es posible realizar tratamientos con sobredentaduras colocando tres implantes de longitudes menores $(7 \mathrm{~mm})$, consiguiendo tasas de éxito comparables'. 


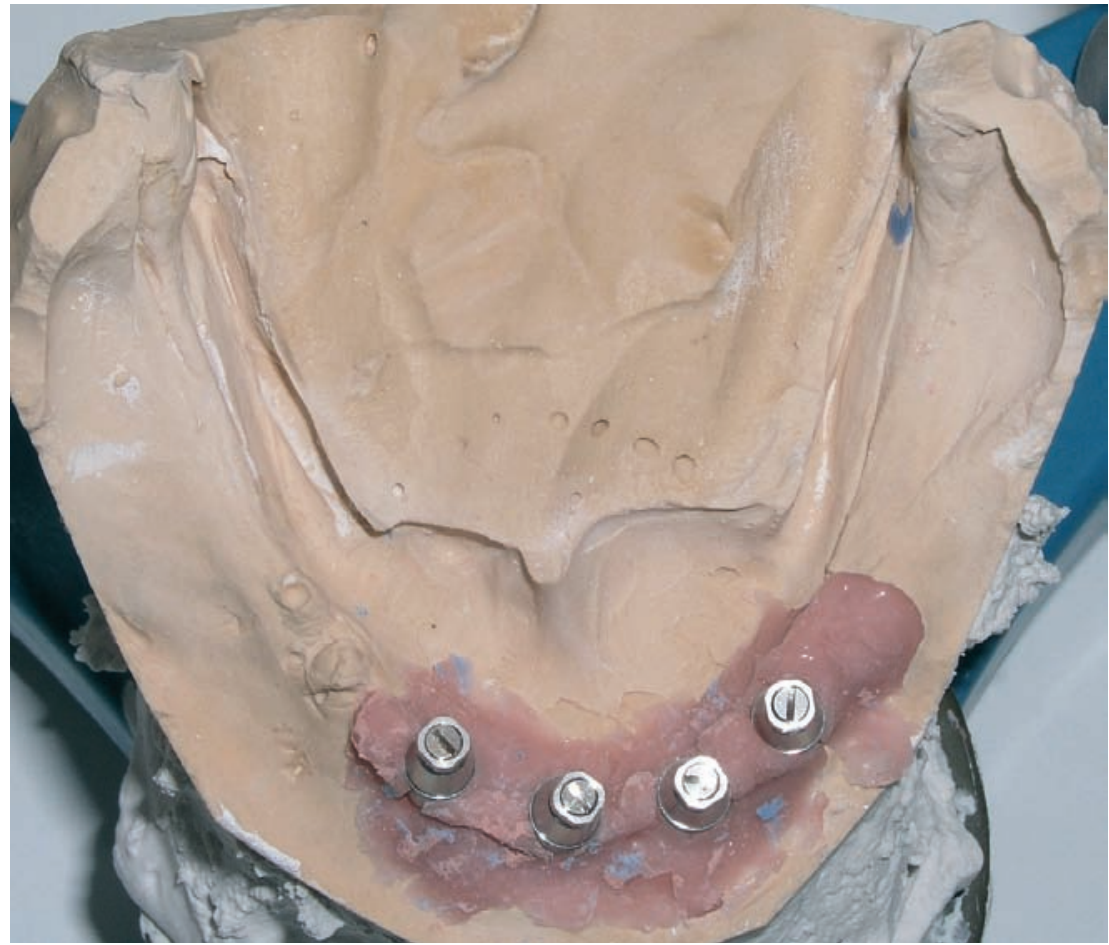

Figura 2. A pesar de que hay cuatro implantes (se perdió el quinto y el paciente no quiso reponerlo), la sobredentaura será de soporte mixto ya que el extremo libre distal en un lado es demasiado largo.

Si nos planteamos realizar una sobredentadura mandibular con carga inmediata, los protocolos más actuales aconsejan la colocación de al menos cuatro implantes ferulizados con una barra $2^{2 *}$. Para el éxito de la prótesis en estas situaciones será necesario que se trate de un hueso de buena calidad (tipos 1 a 3 según la clasificación de Lekholm y Zarb) y conseguir estabilidad primaria en la colocación de los implantes.

En el maxilar superior pondremos siempre al menos cuatro implantes ya que la calidad del hueso es peor que en la mandíbula y, a la vez, la cantidad es menor debido a la presencia del seno maxilar. Cuando la sobredentadura vaya a ser implantomucosoportada será suficiente con estos cuatro implantes, mientras que si queremos realizar una prótesis implantosoportada deberemos poner cinco o mejor seis implantes bien distribuidos. Desde el punto de vista de la carga inmediata, se considera que su aplicación en sobredentaduras maxilares está aún en fase experimental y por tanto no se pueden establecer criterios de aplicación clínica ${ }^{2 * *}$.

La clave que marca la biomecánica en ambas arcadas es que, cuando se pretenda una sobredentadura implantosoportada, será imperativo disponer de implantes tanto en el sector anterior como en ambos sectores posteriores y, además, que la distancia existente entre el implante más distal y el más anterior en ambos lados sea igual o mayor que la longi- tud del extremo libre respectivo. Siempre que se cumpla este precepto, la prótesis será implantosoportada. Cuando no se cumpla en uno o ambos lados, se tratará de una sobredentadura que biomecánicamente se comportará como una prótesis con soporte mixto. Si la barra lleva cantilevers esa distancia se medirá desde el implante más anterior hasta el extremo de cada cantilever. Esto es válido incluso en una arcada superior en la que solamente hay cuatro implantes pero están bastante separados y si los uniéramos con una barra, ésta recorrería buena parte del arco.

\section{Sobredentadura-prótesis<smiles>[101In]</smiles>

Cuando se plantea el tratamiento en el maxilar superior, hay situaciones en las que aún habiendo implantes suficientes para realizar una prótesis fija es preferible optar por una sobredentadura. Normalmente, la decisión de realizar una sobredentadura o una prótesis fija suele enfocarse en función del número de implantes que tengamos a nuestra disposición. Cuando hay cuatro o menos implantes éstos no serán capaces de dar soporte por sí solos a la prótesis y realizaremos una sobredentadura. Cuando el paciente sea portador de cinco o más implantes de suficiente longitud (más de $10 \mathrm{~mm}$ ) y bien distribuidos podremos optar por una prótesis fija totalmente implantosoportada. Ahora bien, hay situaciones en las que, a pesar de disponer de un número suficiente de implantes para realizar una prótesis fija, será preferible 
realizar una sobredentadura. Los principales factores que determinarán nuestra decisión son los siguientes:

- Necesidad de soporte labial. La estética facial se ve afectada cuando no hay el adecuado soporte para el labio. El labio se apoya básicamente en el reborde alveolar y en los cuellos de los dientes anterosuperiores (fig. 3). Las situaciones que conllevan una desdentación prolongada del maxilar superior se acompañan de una reabsorción ósea tanto en sentido craneal como en sentido palatino (fig. 4). Esto es más acentuado si el antagonista es una clase I de Kennedy. La colocación de los implantes en posición lingualizada respecto a la mandíbula a la vez que inclinados tendrá como consecuencia que, si queremos dar el adecuado soporte al labio con una prótesis fija, deberemos crear un voladizo importante para alcanzar el labio. Esta solución será biomecánicamente contraproducente a la vez que favorecerá el depósito de restos alimenticios (fig. 5). Con una sobredentadura será más fácil subsanar ambos problemas. Las clases III de Angle suponen una situación similar de pérdida de soporte labial y de discrepancia entre los dientes anteroinferiores y la emergencia de los implantes maxilares.

- Distancia intermaxilar. Cuando se realiza una prótesis fija implantosoportada y la distancia intermaxilar es excesiva, los dientes de la prótesis serán demasiado largos y pueden quedar espacios amplios entre ellos. Las consecuencias serán problemas de tipo estético, si la sonrisa es gingival, y problemas de tipo fonético al escaparse el aire entre los dientes. Así pues, será difícil reponer tanto los tejidos blandos como los dientes. Se ha

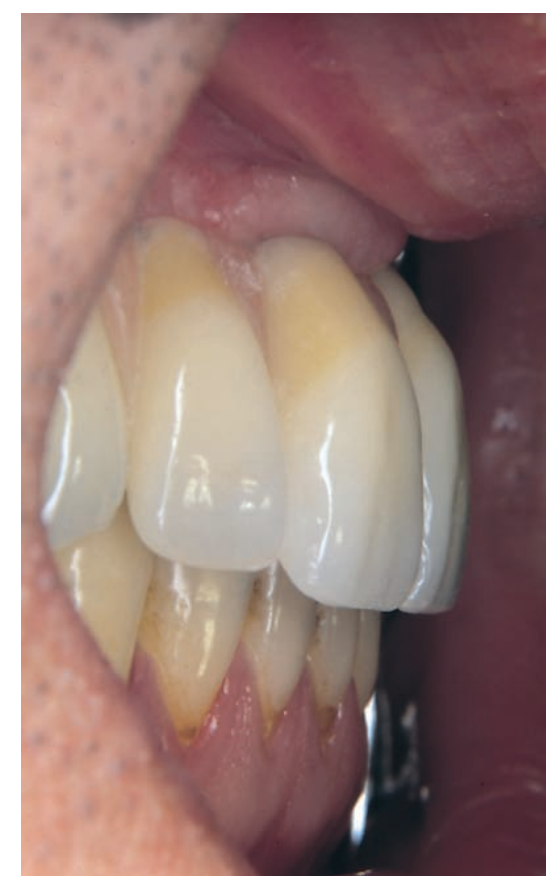

Figura 3. El soporte del labio superior viene dado por el cuello de los dientes anterosuperiores mientras que es la cresta alveolar la que da soporte al filtrum.

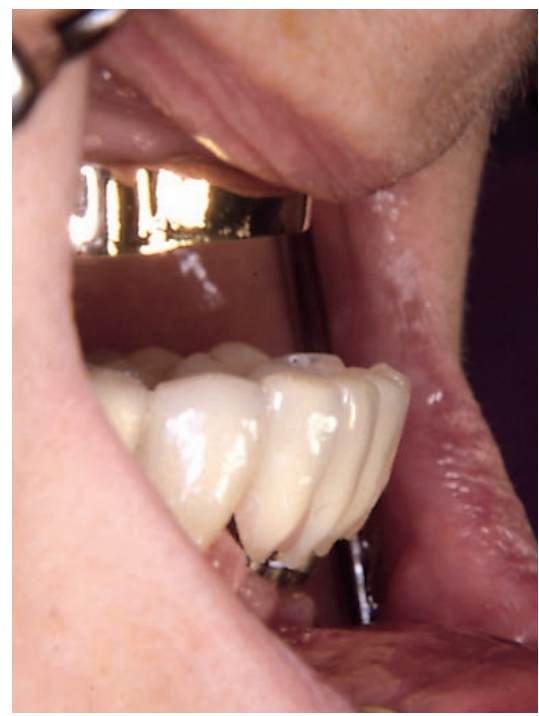

Figura 4. Paciente con una gran discrepancia anteroposterior entre el maxilar y la mandíbula debida a la reabsorción progresiva del proceso alveolar.

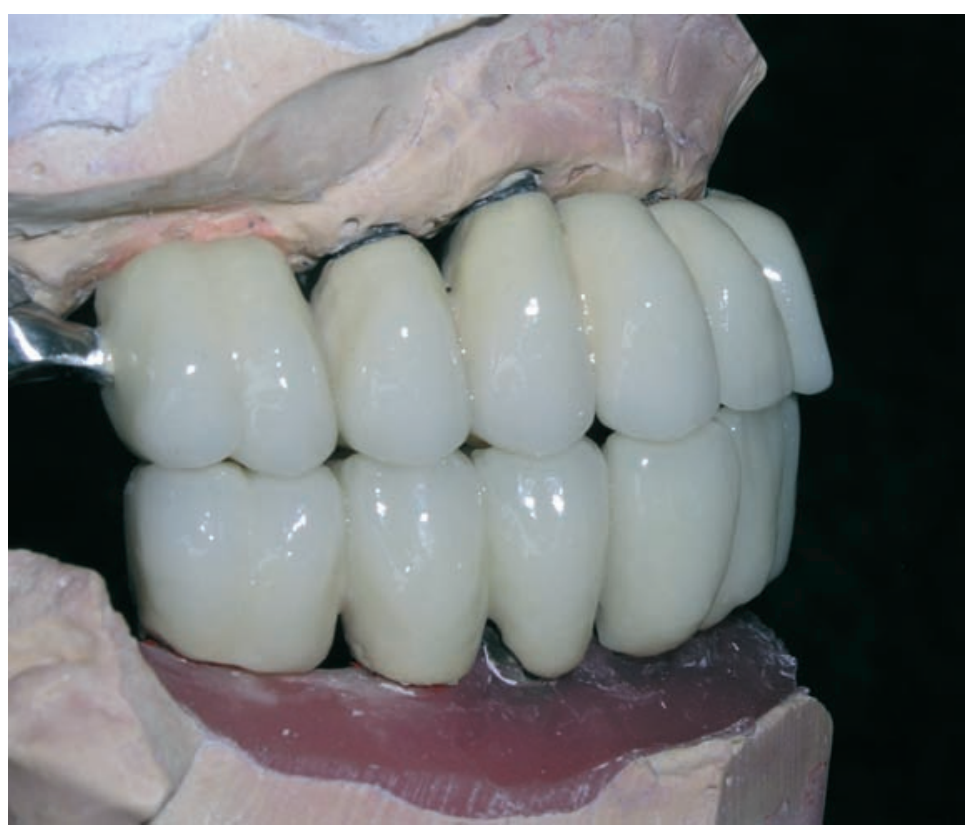

Figura 5. El principal inconveniente de resolver mediante prótesis fija una discrepancia maxilomandibular anteroposterior es la tendencia al cúmulo de alimentos por cervical de los dientes anterosuperiores. 
establecido que el límite se halla en los $15 \mathrm{~mm}$ de distancia entre los bordes incisales de los dientes anteroinferiores y la cresta maxilar, tomando como referencia la dimensión vertical de oclusión ${ }^{3}$. Si la distancia es mayor, será preferible realizar una sobredentadura.

- Higiene de la prótesis. Este es un factor que no hay que subestimar tal y como Feine y cols ${ }^{4 *}$ demostraron en su estudio. En él, el mismo grupo de pacientes recibió en tiempos diferentes los dos tipos de tratamiento, una prótesis fija implantosoportada y una sobredentadura sobre implantes. Pasado el periodo de observación se les preguntó qué opción preferían y el $50 \%$ optaron por la sobredentadura, a pesar de tratarse de una prótesis extraible. La eficacia masticatoria con ambos tipos de prótesis fue considerada similar. La razón que esgrimían era que la higiene era mucho más fácil con la sobredentadura mientras que la higiene de la prótesis fija implantosoportada exigía mayor pericia si se quería realizar adecuadamente. Estos resultados demuestran que una prótesis fija no tiene que ser siempre nuestro objetivo en el tratamiento de un maxilar sin dientes.

- Coste económico. El coste de una prótesis fija siempre es superior al de una sobredentadura y este aumento implica sobretodo a la parte de laboratorio, a la fase clínica y a los propios componentes de los implantes. La diferencia es mayor aún cuando es implantomucosoportada que no cuando es implantosoportada ya que el número de implantes es mayor. La parte quirúrgica puede ver afectado su coste siempre que sea necesario recurrir a técnicas suplementarias como eleva- ciones de seno o injertos o cuando se requiera ingreso hospitalario (p.e si hay que poner implantes pterigoideos o realizar injertos de la cadera).

Por tanto, antes de decidirnos por un tipo u otro de prótesis será necesario contemplar los factores citados. Hay que hacerlo antes de iniciar el tratamiento ya que de lo contrario podemos no cumplir las expectativas del paciente que, esperando un tratamiento fijo puede encontrarse con una estética defectuosa que sólo podrá ser resuelta con una sobredentadura.

\section{Tipo de anclaje- ferulización}

La elección del tipo de anclaje va estrechamente ligada a la determinación de ferulizar o no los implantes. Por lo tanto trataremos las dos cuestiones en el mismo apartado dado que los razonamientos esgrimidos a favor de la ferulización igualmente lo son a favor de las barras y ocurrirá de la misma manera con la no ferulización y los anclajes axiales.

Se argumentó en su momento que la ferulización de los implantes distribuía mejor y más ampliamente las cargas entre los pilares y que reducía el riesgo de aflojamiento de los tornillos así como de fractura de los componentes. Posteriormente, la mayor parte de autores han hallado que los anclajes axiales transmiten las cargas principalmente a la mucosa quedando los implantes liberados de buena parte de ellas, mientras que con las barras aumentan las cargas sobre los pilares ${ }^{5,6}$. También ocurre 10 mismo cuando se produce la desadaptación de las bases como consecuencia de la progresiva reabsorción de la cresta ósea $^{6}$. Si se valoran los estudios realizados sobre el mantenimiento de las sobredentaduras, se observa que el aflojamiento de tornillos es más frecuente cuando se utilizan los anclajes de bola que no cuando son de barra ${ }^{7 *}$. Por último, no se han hallado prácticamente diferencias en cuanto a la supervivencia de los implantes, índice de sangrado, acúmulo de placa y/o pérdida de hueso alrededor de los implantes ${ }^{8}$.

La fuerza retentiva que aportan no es un factor a tener en cuenta cuando se comparan anclajes axiales y anclajes a barra ya que ambos aportan la suficiente retención excepción hecha de los anclajes magnéticos. Aún así, los diferentes estudios comparativos han llegado a la conclusión de que las barras son más retentivas ${ }^{* * 9}$. Por lo que respecta a los anclajes magnéticos, son con diferencia los menos retentivos, los que aportan menor estabilidad a la prótesis y menos comodidad durante la masticación, a la vez que las complicaciones son, de forma clara, más frecuentes ${ }^{9}$. Por ello, considera el autor que son totalmente desaconsejables por cuanto existen alternativas mejores.

La biomecánica también es un criterio fundamental en la elección del sistema de anclaje, pero tanto las barras como los anclajes axiales admiten situaciones tanto de soporte exclusivamente implantario como situaciones de soporte mixto. En este sentido, debemos tener presente, aunque se trate de sobredentaduras sobre implantes, las afirmaciones realizadas por 


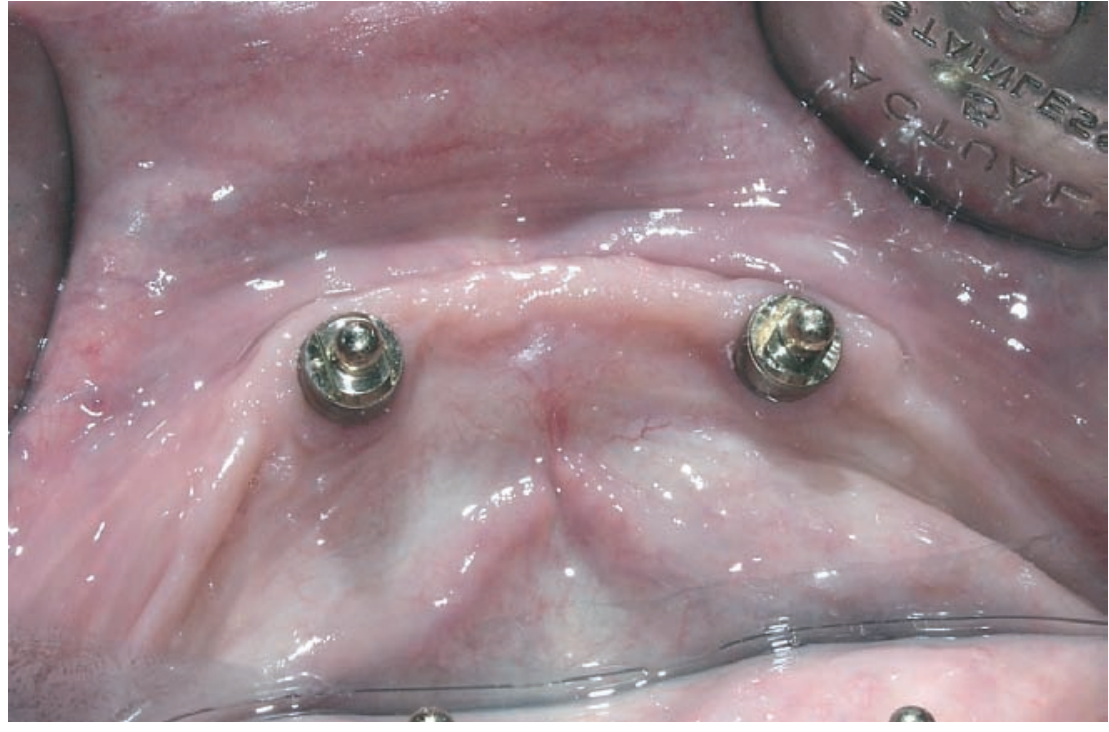

Figura 6. La situación más sencilla a nivel mandibular es la colocación de dos implantes, que generalmente se pueden situar paralelos.

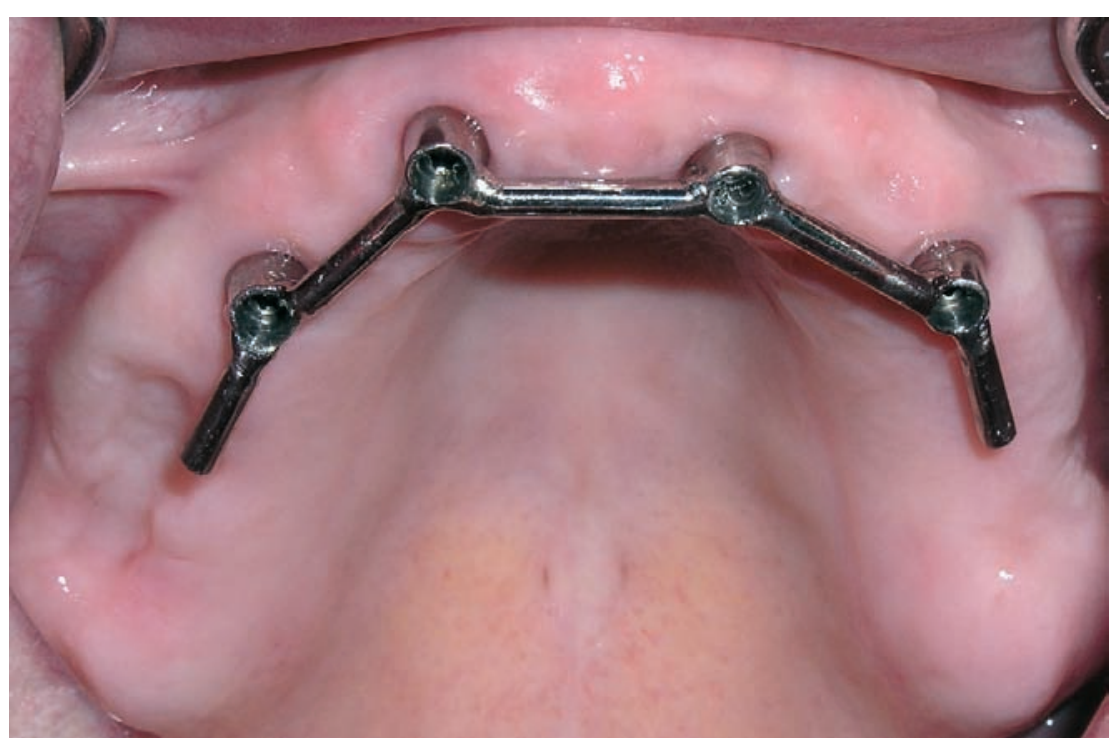

Figura 7. La emergencia divergente de los implantes maxilares se resuelve sin problemas mediante la utilización de barras.

Stewart y Edwards ${ }^{10}$ que siguen siendo vigentes. Consideraban que es el diseño del anclaje el que realmente determina las repercusiones sobre los pilares y los tejidos de soporte, y que la sobrecarga aparecerá con mayor probabili- dad en aquellos diseños de anclajes en los que haya un contacto íntimo entre el macho y la hembra y no se dote al sistema de resiliencia alguna.

Entonces, iqué factores nos inclinarán a decidirnos por un anclaje axial o una barra? Los enumeraremos a continuación:

- Mandíbula o maxilar superior. En la mandíbula siempre será más factible utilizar anclajes axiales ya que la colocación de los implantes paralelos o casi paralelos es mucho más probable (fig. 6). Incluso si el número de implantes es de cuatro o cinco podemos conseguir un relativo paralelismo, aunque si no es así la alternativa será una barra. Las casas comerciales nos dan cierto margen de tolerancia ya que este tipo de anclaje admite habitualmente discrepancias de hasta $20^{\circ}$ entre los implantes, aunque hay una versión del Locator ${ }^{\circledR}$ de Zest Anchors que es capaz de admitir discrepancias de hasta $40^{\circ}$. En el maxilar superior, la emergencia claramente divergente de los implantes, el hueso de peor calidad y en menor cantidad y la utilización de implantes con frecuencia más cortos condicionará la utilización de barras, independientemente de la biomecánica de la prótesis (fig. 7).

- Forma de la arcada. Es idóneo colocar la barra sobre la cresta con el fin de no ocupar el espacio destinado a la lengua ni condicionar grosores importantes en la zona anterior del paladar dificultando la fonética. También se ha afirmado que es aconsejable colocar la barra paralela al eje de rotación mandibular, aunque no hay evidencia científica de ello. En las arcadas cuadradas es posible cumplir ambos requisitos, pero en las arcadas estrechas es más probable que la barra realice su recorrido por fuera de la cresta por lo que será preferible utilizar anclajes axiales. Una alternativa podría ser crear un voladizo con la barra, pero esta situación generaría 
brazos de palanca indeseables sobre los pilares.

- Espacio interarcada. Los anclajes axiales son más bajos y no requieren tanto espacio como las barras. Cuando nos planteemos utilizar barras debemos contar con $1 \mathrm{~mm}$ que debe quedar entre la barra y la encía, los $2 \mathrm{~mm}$ o incluso $3,5 \mathrm{~mm}$ de la propia barra (según se trate de una barra Ackerman o una barra Dolder), los 0,5-1 mm del espaciador (si es implantomucosoportada), el 1-1,5 mm de la hembra y la resina que la fija y $1 \mathrm{~mm}$ o más según quede por encima sólo la estructura metálica o coloquemos además dientes artificiales. Por tanto, para poner una barra es necesario disponer como mínimo de $6 \mathrm{~mm}$ aunque fácilmente pueden ser más. Es fundamental para determinar el espacio disponible realizar un montaje de dientes en cera y valorar en el articulador si es posible colocar una barra o, por el contrario, el espacio es insuficiente.

- Grado de reabsorción ósea. A pesar de que en su momento se consideraba que cuando la reabsorción ósea era importante era preferible utilizar barras ya que aportaban una mayor estabilidad, si los implantes son suficientemente largos (más de 10 $\mathrm{mm}$ ) es posible conseguir una buena estabilidad con anclajes axiales.

- Longitud de los implantes. Se ha constatado un mayor número de fracasos con implantes menores de 10 $\mathrm{mm}$, sobretodo en el maxilar (hasta un $24 \%$ de fracasos en implantes de $7 \mathrm{~mm}$ según Jemt y Lekholm¹1). Por ello, cuando los implantes tengan longitudes menores de $10 \mathrm{~mm}$ se optará por ferulizar y repartir cargas mientras que si son más largos podremos hacerlos trabajar independientemente. Esto es

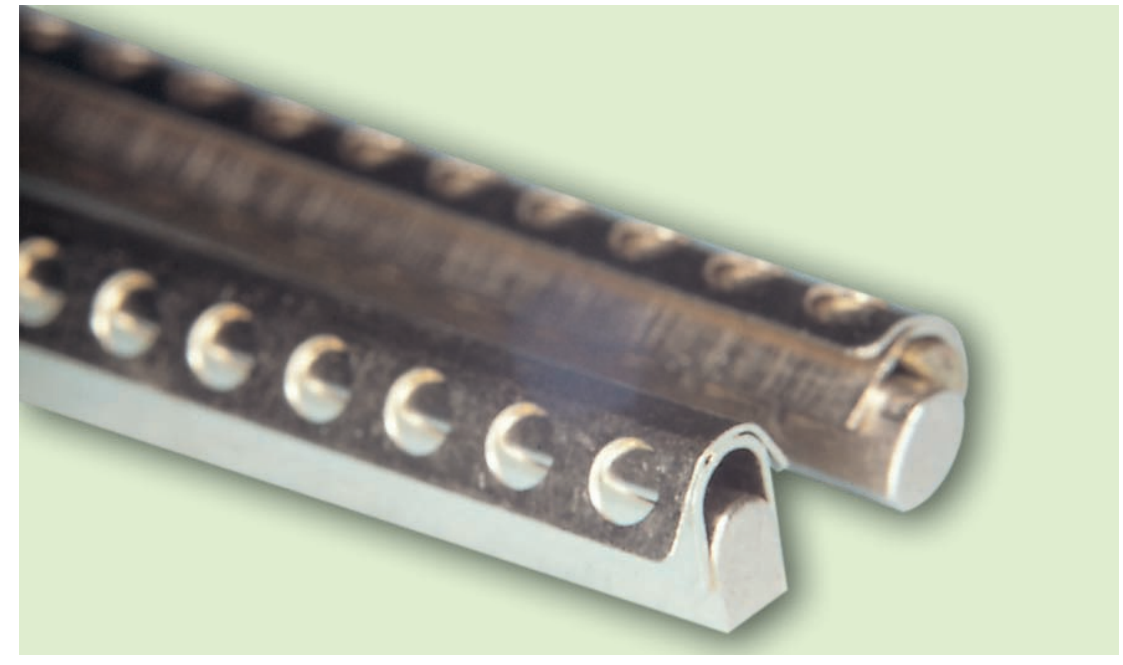

Figura 8. Barras Dolder con los dos distintos tipos de sección, ovoide y en U.

cierto para implantes mecanizados de superficie no tratada por lo que debería valorarse con implantes de superficie tratada u otros tipos, como los implantes Endopore ${ }^{\circ}$.

- Localización de los implantes. No es conveniente utilizar tramos de barra excesivamente largos ya que aumentan las tensiones transmitidas al hueso ${ }^{12}$ a la vez que es más difícil situar la barra sobre la cresta sin crear voladizos. Por ello, nos marcaremos la posición de los caninos como referencia de máxima separación entre implantes (se hallan separados por unos 22-23 mm).

Como podemos comprobar, el análisis de estos factores ya nos inclinará hacia un tipo u otro de anclaje. No entraremos a detallar los distintos tipos de anclajes axiales ya que una vez se ha determinado cuál va a ser el comportamiento biomecánico de la prótesis, la elección entre ellos estará en función del espacio disponible y también de las preferencias personales. En cambio, sí que es conveniente precisar una serie de detalles concer- nientes al diseño de las barras que son importantes:

- Tipo. Disponemos de barras Ackerman y barras Dolder. La barra Ackerman es redonda 1 ,8 $\mathrm{mm}$ de diámetro), colable y con caballitos cortos y simples (unos $5 \mathrm{~mm}$ ), lo que permite adaptarla al contorno de la cresta tanto en sentido vestíbulolingual como en sentido oclusogingival. La barra Dolder puede ser ovoide o en $U$, se presenta en dos alturas distintas (3,5 $\mathrm{mm}$ y $3 \mathrm{~mm}$ ), en dos longitudes distintas (30 y $50 \mathrm{~mm}$ ) y es mecanizada (fig. 8). La hembra es un único y largo caballito, por lo que sólo se utilizará cuando la barra vaya a ser totalmente recta y quede situada sobre la cresta. La barra Ackerman y la barra ovoide pueden ser utilizadas tanto en situaciones de soporte mixto (con espaciador) como en prótesis implantosoportadas (sin espaciador), mientras que las barras en U sólo pueden utilizarse en casos implantosoportados ya que impiden toda resiliencia. Una alternativa a las barras en U son las barras fresadas (figs. 9a y 9b). 

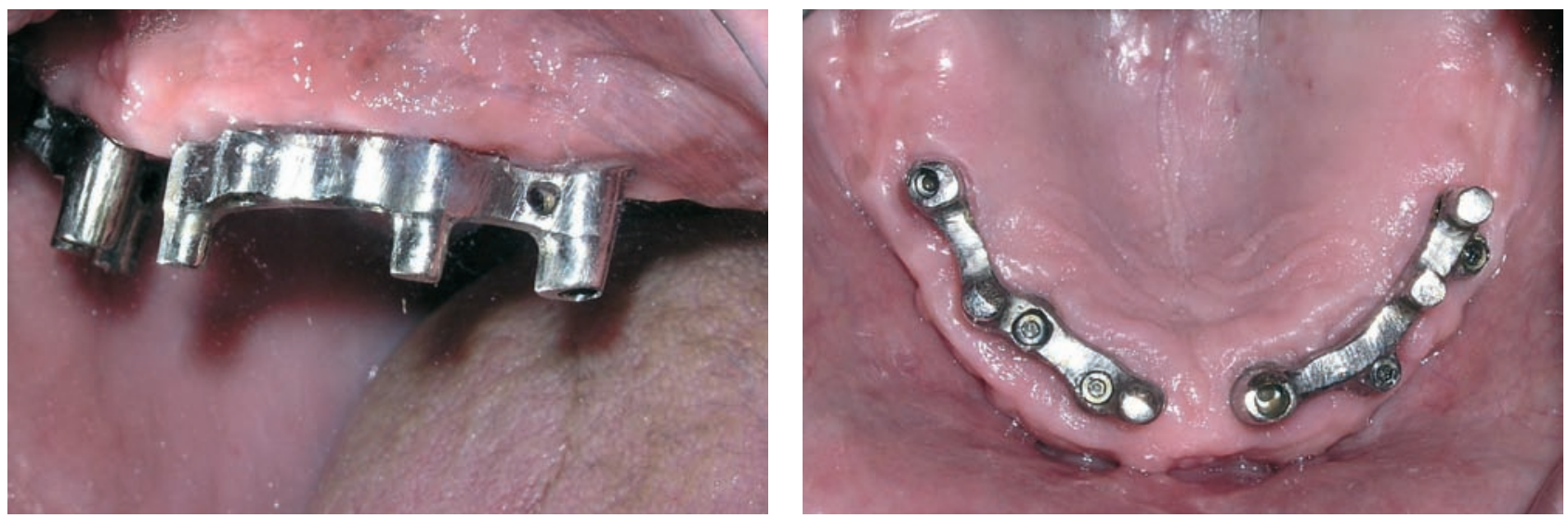

Figura 9A y 9B. Paciente tratado con una sobredentadura maxilar implantosoportada sobre seis implantes. Se realizaron dos barras fresadas de titanio con pasadores sobre las que encajaba la estructura de la sobre dentadura.

- Orientación. Aunque siempre se ha afirmado que es deseable que sea paralela al eje de rotación mandibular no se han investigado los efectos de no cumplir con esta premisa. Lo que está claro es que sólo es factible cumplirlo cuando se trabaja sobre dos implantes situados de forma simétrica - casi simétrica a ambos lados de la línea media. Cuando no se da esta situación difícilmente conseguiremos que la barra sea en su totalidad paralela al eje de rotación mandibular. En su momento, cuando se planteaba el tratamiento con sobredentaduras implantomucosoportadas mandibulares se aconsejaba poner tres implantes por si acaso uno fallaba. De esta manera, difícilmente se podía conseguir que toda la barra cumpliera el paralelismo. Actualmente, como se considera suficiente colocar sólo dos implantes para este tipo de tratamiento es más fácil alcanzar ese objetivo.

- Situación. Debemos situar la barra sobre la cresta. Con ello se persigue evitar invadir el espacio de la lengua, engrosar en exceso la prótesis superior para no interferir con la fonética, dificultar la colocación de los dientes artificiales y eliminar voladizos de la barra que sobrecargarían los implantes. Igualmente, es fundamental facilitar la higiene debajo de la barra, por ello dejaremos 1-2 mm de espacio por debajo de ella. Este espacio facilita la hiperplasia gingival por lo que en un tiempo se aconsejó soldar unas proIongaciones metálicas que fueran de la barra a la encía y ocuparan ese espacio (barra Hader). El problema radicaba en que se dificultaba aún más la higiene y tampoco se evitaba el acúmulo de placa bacteriana ni de tártaro, por lo que actualmente se prefiere dejar ese espacio libre.

- Longitud. Los tramos entre pilares deberán ser de al menos unos $10 \mathrm{~mm}$ (para que quepan los caballitos de la barra Ackerman que miden unos 5 $\mathrm{mm}$ ) e inferiores a $23 \mathrm{~mm}$ para no incrementar las tensiones transmitidas al hueso ${ }^{12}$. Si tienen que ser mayores de $23 \mathrm{~mm}$ será mejor optar por una barra fresada ya que tiene un calibre mayor, es mucho más rígida y no flexionará o también realizarla en dos tramos independientes (figs.10a y 10b).

- Tramos a extensión distal. Debemos ser cautelosos con los tramos a extensión distal o cantilevers ya que su presencia se asocia a una mayor sobrecarga del implante más distal, en particular si no ajustan correctamente las bases a la mucosa $a^{3,6}$. En su momento se indicaron para aportar estabilidad a las sobredentaduras implantomucosoportadas. Pero no tiene sentido utilizarlos para tal fin ya que la estabilidad sólo se consigue a través de una íntima adaptación del cantilever a la sobredentadura, algo que en una prótesis implantomucosoportada no es deseable ya que se sobrecargarán los implantes. Si a pesar de todo se quieren utilizar, no sobrepasarán los $7 \mathrm{~mm}$, tal y como se observa en la fig. 11, y la suma global de ambos no superará la longitud de la barra que une ambos implantes. Además, Ios inclinaremos hacia gingival para evitar que, con la reabsorción progresiva de la cresta, se acabe apoyando la prótesis en ellos y provoque el aflojamiento de tornillos o incluso la fractura del propio cantilever ${ }^{13}$. De hecho, den Dunnen y cols $^{14}$ hallaron una mayor frecuencia de fracturas de las barras en aquellas que llevaban cantilevers $y$, además, todas las fracturas se produ- 


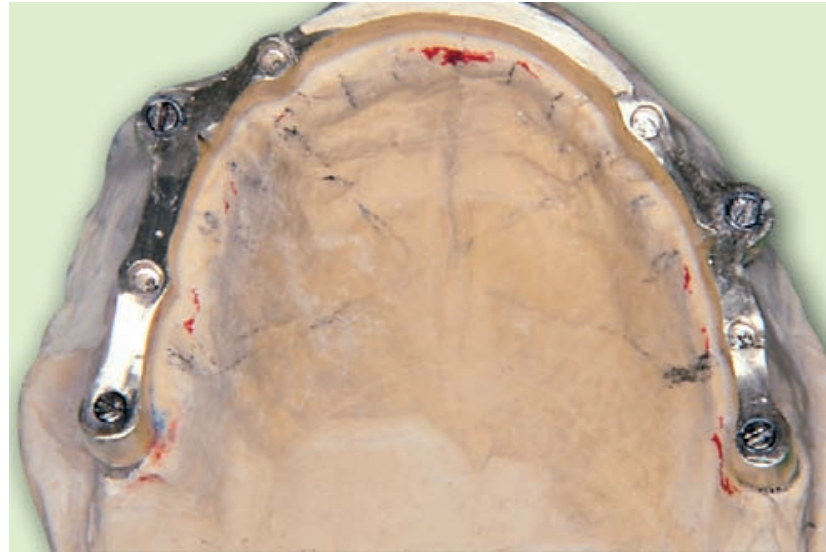

Figura 10A. Barra fresada maxilar sobre cuatro implantes. Se crea un gran voladizo anterior que generará brazos de palanca que actuarán sobre los implantes más distales, actuando de fulcro los implantes más anteriores.

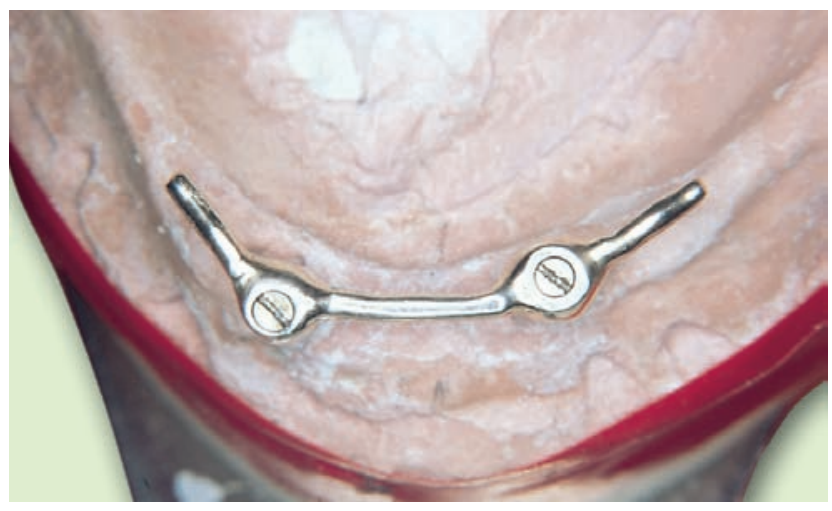

Figura 11. Cuando se utilicen cantilevers, estos no superarán los $7 \mathrm{~mm}$ de longitud.

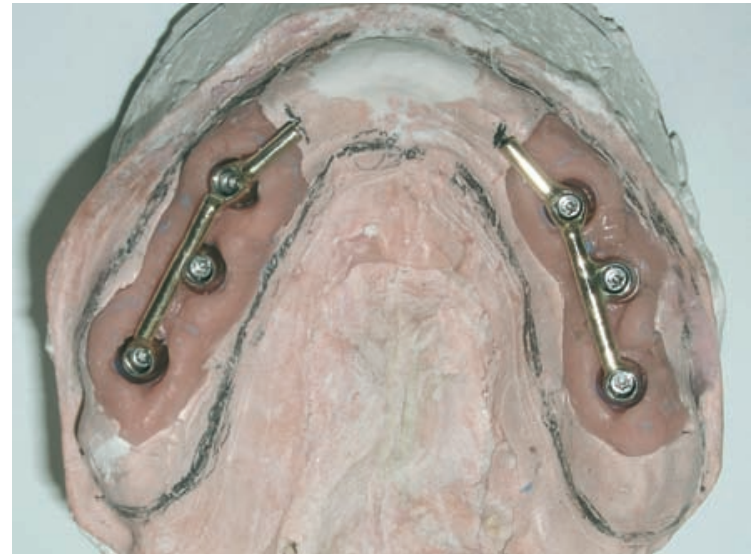

Figura 10B. Paciente tratado con dos barras Ackerman independientes. Es preferible no crear voladizos y trabajar en estos casos con dos barras independientes ya que la biomecánica de

la sobredentadura será la misma (implantosoportada), no habrá brazos de palanca y no se dificultará el montaje de los dientes anteriores

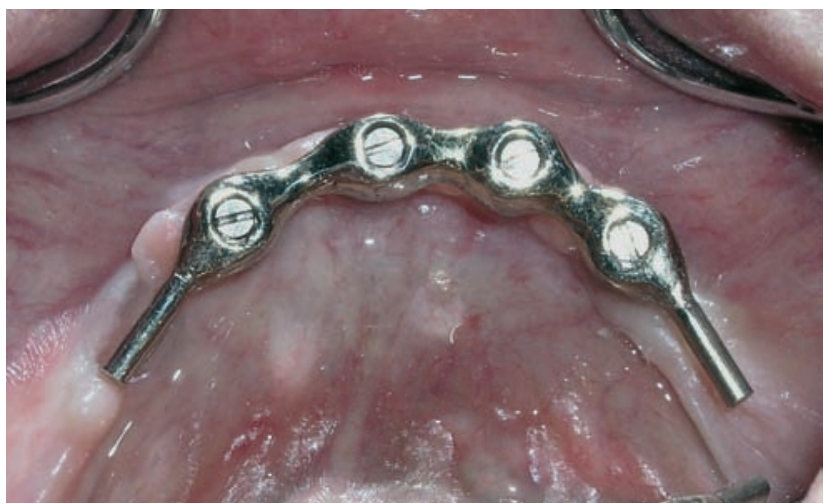

Figura 12. Cuando los implantes estén excesivamente juntos se colocarán los caballitos en los cantilevers. cían a nivel de estos. En principio, será preferible no situar los caballitos en ellos ya que aumentan las tensiones sobre el implante adyacente ${ }^{6}$ pero cuando los implantes estén excesivamente juntos careceremos del espacio suficiente para los caballitos por lo que la barra tendrá cantilevers y los colocaremos sobre ellos (fig.12). Una alternativa a este diseño con cantilevers puede ser la colocación de anclajes tipo miniDalbo o bolas Roach en los extremos de las barras. En conclusión, desde el punto de vista de la biomecánica, cuando se trate de una prótesis implantomucosoportada no se utilizarán cantilevers siempre y cuando tengamos espacio entre los implantes para colocar los caballitos; en cambio, cuando se trate de una prótesis implantosoportada podrán utilizarse con garantías.

Por último, hay un aspecto importante que concierne a los anclajes y es el referente a la fijación de los mismos a la sobredentadura. Cuando se trata de una prótesis implantosoportada será preferible la fijación de los mismos en el laboratorio. No se utilizarán espaciadores ya que se busca el contacto íntimo entre la prótesis y el anclaje. En cambio, cuando se trate de una sobredentadura implantomucosoportada es más aconsejable fijar los anclajes directamente en boca ya que es la manera más segura de 


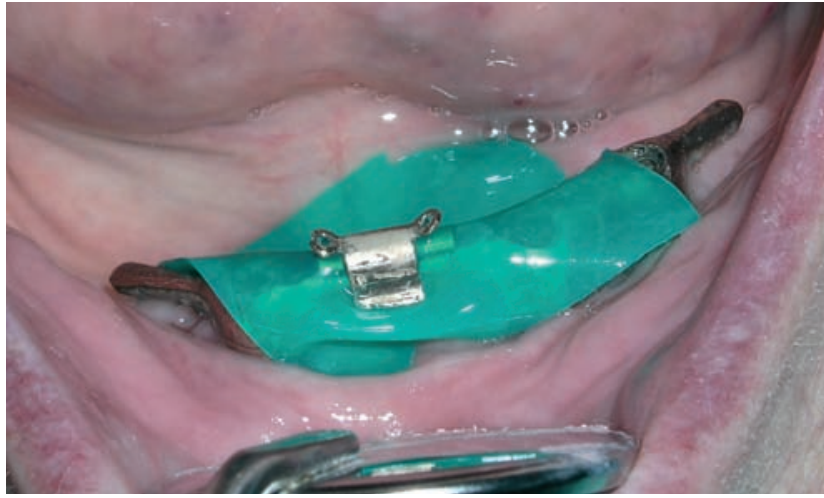

Figura 13. Se observa la fijación en boca de un caballito a una sobredentadura implantomucosoportada. Es fundamental colocar un espaciador entre él y la barra con el fin de dotar de resiliencia al sistema.

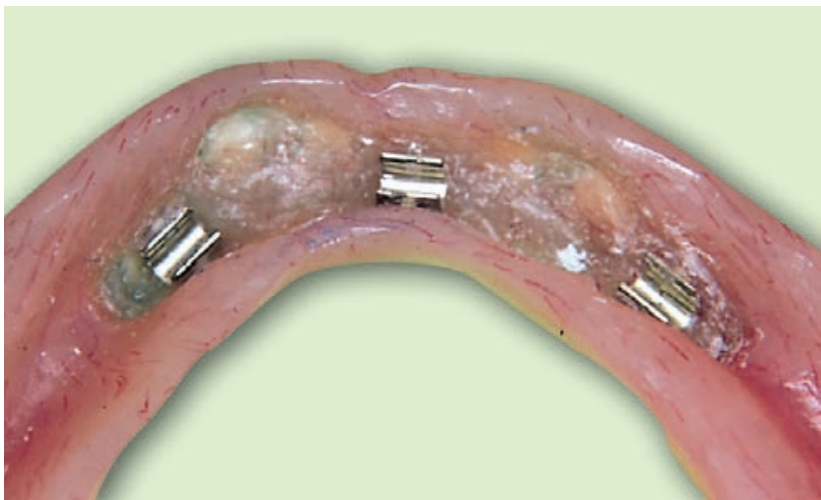

Figura 14. La zona de la sobrdentadura que recubre los pilares y contiene los anclajes presenta un grosor de acrílico significativamente menor, por lo que será una zona de baja resistencia. garantizar el alivio generado por el espaciador. En estos casos, siempre colocaremos espaciadores para evitar el contacto directo entre el anclaje y la prótesis y dotarla así de resiliencia (fig.13). El problema reside en que algunos sistemas de implantes suministran anclajes que no contemplan el uso de espaciadores con lo que desde el primer momento el contacto es íntimo (sobretodo anclajes axiales). Con la reabsorción progresiva de la cresta ósea aumentará la carga sobre los implantes. Cuando en las citas de revisión se plantee realizar rebasados, siempre los haremos colocando el espaciador entre el macho y la hembra para volver a dotar de alivio debajo del caballito.

\section{Estructura colada de refuerzo}

Hay dos factores que nos aconsejan reforzar las sobredentaduras mediante una estructura colada:

- La reabsorción progresiva de la cresta ósea debajo de las sobredentaduras implantomucosoportadas favorece que éstas se apoyen sobre los pilares. Éstos actuarán a modo de cuña debajo de la prótesis induciendo la aparición de fisuras y fracturas en el acrílico. Hay que tener presente que es necesario realizar rebasados de forma periódica ( $24 \%$ al año según Jemt y col $^{15}, 44 \%$ a los dos años según Smedberg y $\mathrm{col}^{16}$ ) y que, con frecuencia, los pacientes no acuden a las citas de revisión o lo hacen más tarde de cuando debieran.

- Independientemente de la biomecánica de la prótesis, la zona de la sobredentadura que está ocupada por el sistema de pilares y anclajes presenta un grosor de acrílico significativamente menor, por lo que será una zona de baja resistencia (fig. 14).

Las consecuencias de ambos factores quedaron demostradas por Langer y Langer ${ }^{17}$, que valoraron la presencia de fracturas en sobredentaduras sin refuerzo de pacientes geriátricos. Colocaron dieciocho sobredentaduras, de ellas doce eran mandibulares y seis maxilares. En el periodo que va de los cuatro meses postinserción a los dos años, nueve de las doce prótesis inferiores se fracturaron, mientras que en el periodo que va de los once meses postinserción a los cuatro años, se fracturaron la mitad de las prótesis superiores. Así como las fracturas de las prótesis mandibulares se produjeron independientemente de que el antagonista fueran dientes naturales o dientes artificiales, en el caso de las prótesis maxilares el antagonista siempre presentaba dentición natural. Pero lo que es más importante, en todos los casos y sin excepción, las fracturas se situaban a nivel de los pilares. Si bien se trataba de un estudio de sobredentaduras sobre dientes, los resultados y conclusiones son totalmente aplicables a las sobredentaduras sobre implantes ya que mecánicamente se comportan igual.

La utilización de una estructura metálica nos permite:

- Reforzar la base de acrílico evitando la fractura de la sobredentadura, sobretodo en aquellas situaciones en las que el antagonista es la dentición natural. 


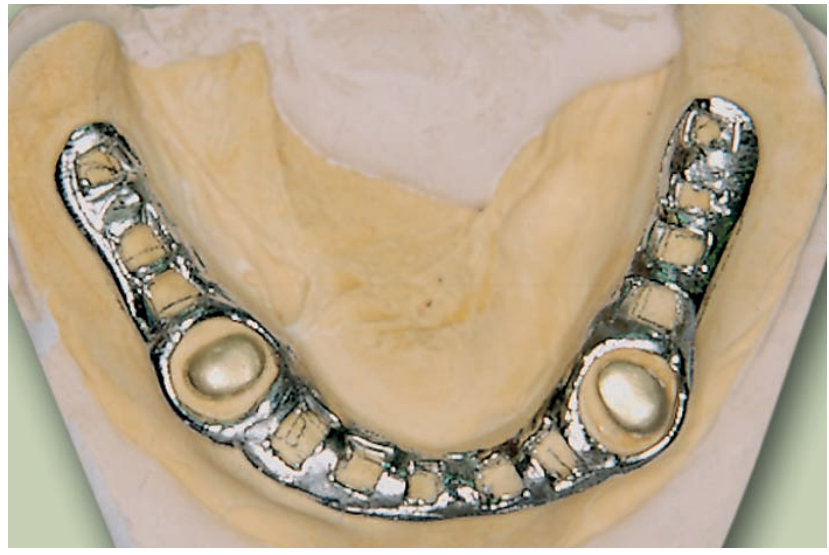

Figura 15A. Estructura de Cr-Co mandibular en escalera. El armazón no presentará elementos que crucen por encima de los pilares ya que en estas zonas el espacio disponible suele ser reducido.

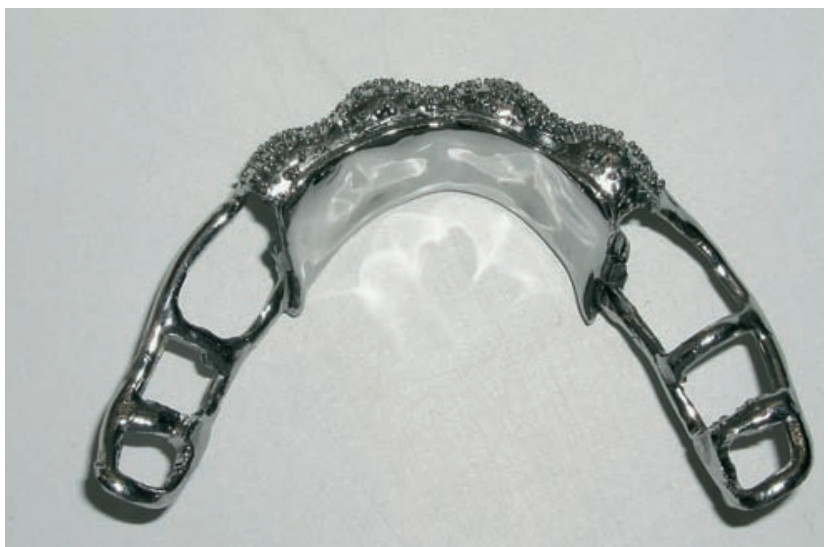

Figura 15B. Una alternativa cuando el espacio vestíbulolingual sea escaso será colocar una placa lingual metálica.

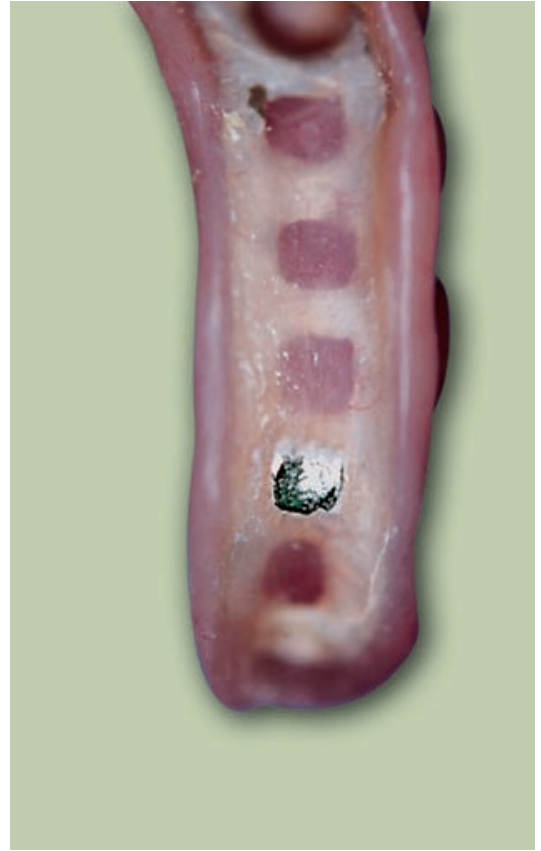

Figura 16. Se colocarán topes tisulares anteriores y posteriores con el fin de evitar el desplazamiento de la estructura de

$\mathrm{Cr}$-Co en el momento del empaquetamiento de la resina.

- Reducir el grosor de la prótesis por lingual o por palatino dejando más espacio para la lengua.

- Aumentar la supervivencia de la

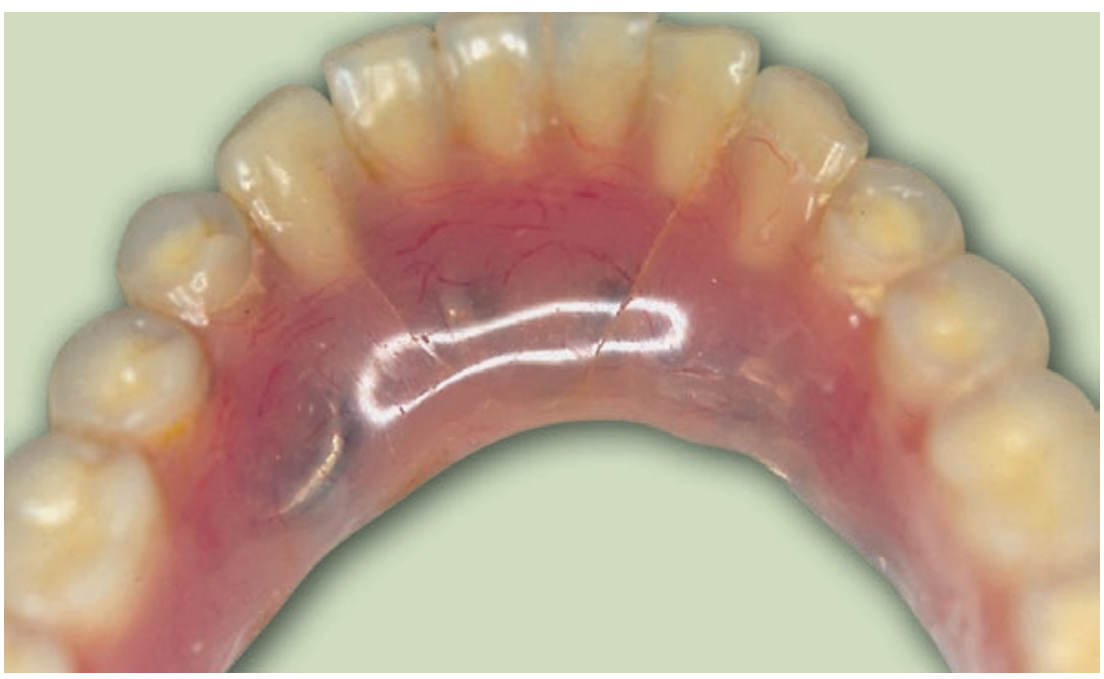

Figura 17. Sobredentadura mandibular en la que se observa la presencia de dos fisuras en la zona de pilares. De no ser por la estructura metálica ya se habría producido la fractura.

sobredentadura sin incrementar de forma significativa el coste de la misma.

Por tanto, toda sobredentadura llevará incluida una estructura de $\mathrm{Cr}$ Co cuyo diseño será similar al de las bases de prótesis parcial removible (figs.15a y 15b), en escalera, con una barra lingual y otra vestibular unidas por barras transversales y con sus topes tisulares ${ }^{13}$. Los topes estarán bien distribuidos para evitar el desplazamiento de la estructura hacia gingival durante el empaquetamiento de la resina (fig.16) y se evitará que crucen elementos de la misma por encima de los anclajes puesto que allí el espacio es más reducido. Y aún así, a veces serán patentes las fisuras en el acrílico producidas por la situación descrita, aunque no se llegue a fracturar la 


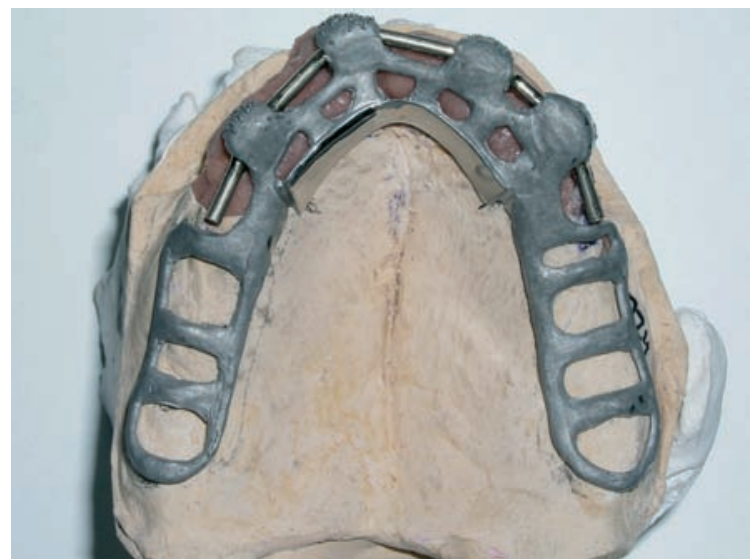

Figura 18. Estructura maxilar en la que los implantes aportan toda la retención.

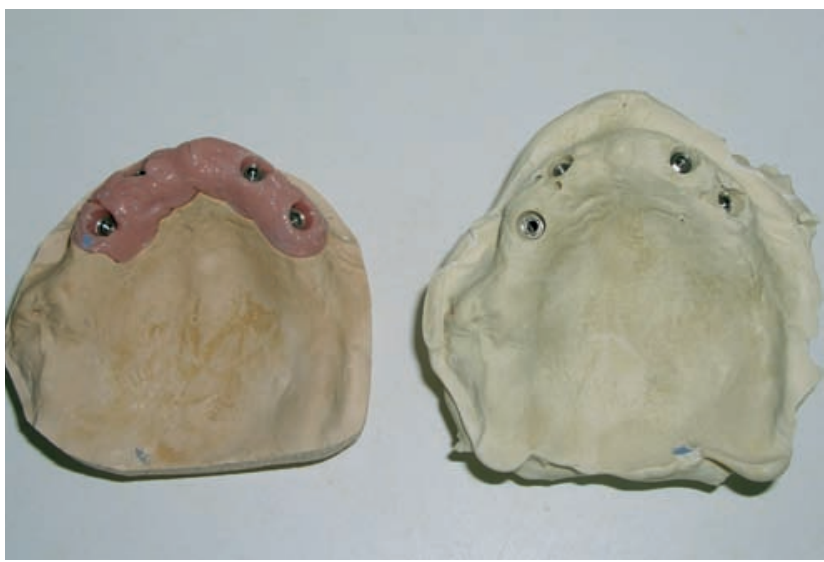

Figura 19. Dos modelos del mismo paciente, uno con encía blanda para fabricar la barra y la sobredentadura y el otro, sólo con escayola, para realizar el sellado periférico. sobredentadura. Esto es más evidente en las prótesis inferiores que no en las superiores ya que la superficie mucosa de apoyo es menor. En la figura 17 podemos observar la presencia de dos fisuras en una sobredentadura mandibular que lleva una estructura de refuerzo (de no ser por el refuerzo ya se habría fracturado).

Además, en las sobredentaduras mandibulares implantomucosoportadas la prótesis alcanzará siempre el trígono retromolar que será recubierto sólo por acrílico. La utilización de los trígonos en el soporte de la sobredentadura reducirá la velocidad con que se reabsorberá la cresta ósea a la vez que aportará una mayor estabilidad horizontal.

El diseño de la sobredentadura maxilar superior puede cubrir o no el paladar. La eliminación del recubrimiento palatino tiene un doble efecto sobre la prótesis: por un lado, se reduce la superficie de soporte por lo que se transfiere mayor responsabilidad en el mismo a los propios implantes y, por otro, se pierde la capacidad de sellado periférico y sellado posterior de la sobredentadura con lo que se transfiere toda la responsabilidad a los implantes en lo que a retención se refiere. Palmqvist y $\operatorname{cols}^{18}$ ya sugerían que la ausencia de recubrimiento palatino suponía un aumento del riesgo de fracaso de los implantes en aquellos casos donde hay poco hueso o éste es de mala calidad. Así pues, no será posible eliminar el recubrimiento del paladar a menos que los implantes sean capaces de colaborar en el soporte de forma clara y de aportar toda la retención a la prótesis (fig.18). Si es factible colocar cuatro o más implantes, son de más de 10 mm y no están en situación comprometida podremos dejar libre el paladar con lo que mejorará la comodidad del paciente y se alterará la fonética en menor grado. Si sólo podemos poner dos o tres implantes, cubriremos siempre el paladar y así rebajaremos las exigencias funcionales y mecánicas sobre los mismos.

Se efectúe o no el recubrimiento palatino siempre se realizará un sellado periférico de la prótesis para evitar que penetren restos de alimentos por debajo de la sobredentadura. Para ello se labrará un surco de $1 \mathrm{~mm}$ de anchura y 0,5-1 $\mathrm{mm}$ de profundidad en el modelo en todo el perímetro palatino de la sobredentadura. La profundidad del surco variará según el grosor de la encía, siendo de $1 \mathrm{~mm}$ en aquellas zonas en las que la encía es más gruesa, como ocurre por palatino de los implantes (seguramente por el efecto de succión de la prótesis provisional sobre los tejidos que se desplazaron al realizar el colgajo en el momento de colocar los implantes). Hay que tener presente que si se realiza el vaciado del modelo a nivel de los implantes con resina blanda o silicona rosada no será posible labrar en ella el surco para el sellado periférico. Por ello, deberemos realizar dos vaciados de la impresión definitiva, uno con resina blanda para confeccionar la barra y la prótesis, y otro sólo con escayola para poder realizar el sellado periférico (fig.19).

El refuerzo que se propone para las sobredentaduras también se hace extensivo al antagonista cuando éste es una prótesis completa. La sobrecarga que reciben las prótesis completas 


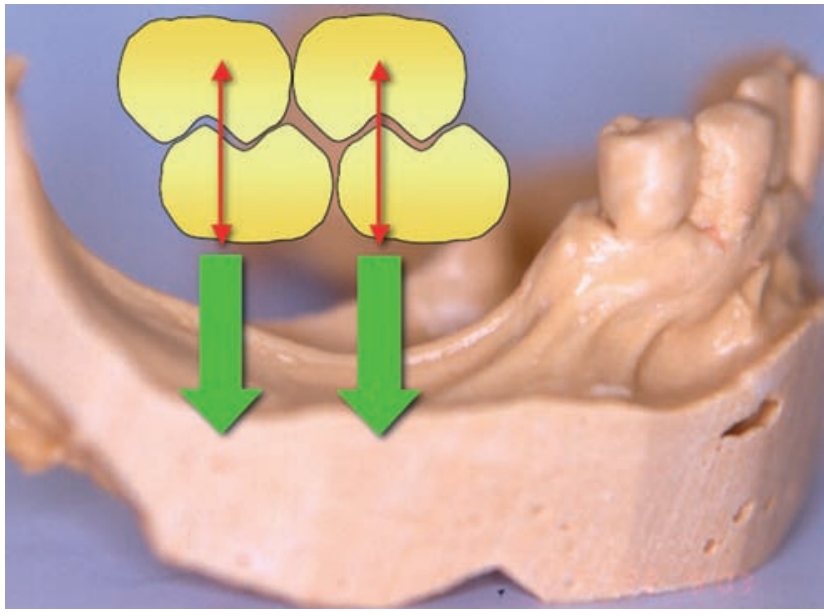

Figura 20. Cuando el paciente ocluye los contactos entre dientes antagonistas estabilizan la prótesis.

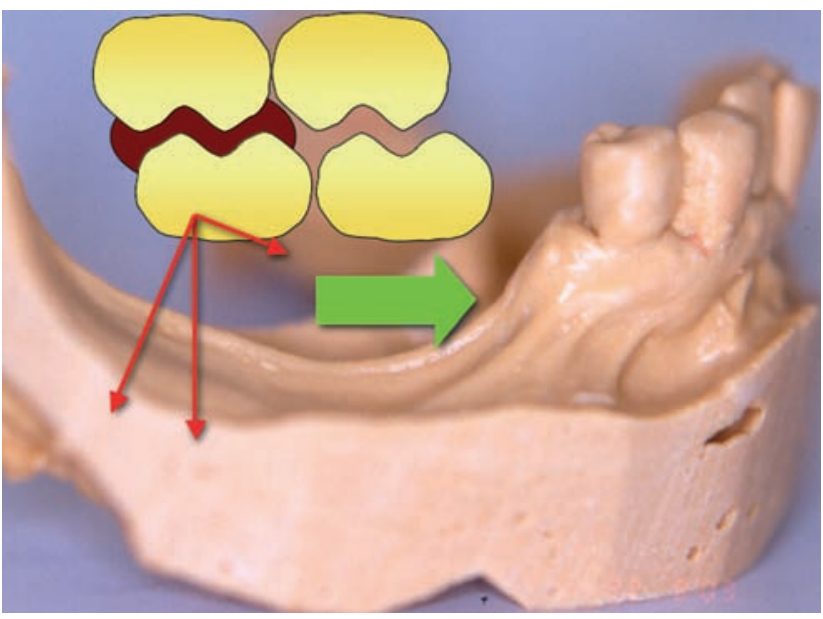

Figura 21. Cuando se interpone alimento desaparece la capacidad estabilizadora de la oclusión y se genera una carga sobre un plano inclinado. La fuerza se descompone en dos, una dirigida hacia la cresta, que será la responsable de la posterior reabsorción, y otra dirigida hacia mesial, que es la potencialmente desestabilizadora.

antagonistas a nivel de la línea media se demuestra con la elevada incidencia de fracturas en las mismas (alcanza el $10 \%$ según Haraldson y cols $^{19}$ ). Este hecho viene potenciado por la reabsorción de la cresta a nivel anterior en lo que es un auténtico síndrome combinado de Kelly, aunque en este caso los pilares son implantes. Han sido varios los autores que han descrito su aparición en pacientes portadores de una sobredentadura sobre implantes mandibular y una prótesis completa superior $^{20,21}$. Para evitar todo este proceso será conveniente, en primer lugar, reforzar la prótesis completa antagonista con una malla metálica o con un paladar de $\mathrm{Cr}$-Co. Además, con el fin de enlentecer la reabsorción será fundamental un correcto ajuste oclusal dejando los dientes anteriores en ligera infraoclusión cuando el paciente ocluya en máxima intercuspidación y consiguiendo una buena oclusión balanceada cuando el paciente realice movimientos excursivos. En último término, lo idóneo sería poder equiparar el soporte maxilar con el de la mandíbula convirtiendo la prótesis completa superior en una sobredentadura sobre implantes. Otra alternativa posible si deben coronarse los dientes anteroinferiores, es utilizar coronas de metal con recubrimiento de resina con el fin de igualar la dureza de éstos a la de sus antagonistas, con lo que el desgaste será similar en ambas arcadas.

\section{Montaje de dientes}

En cuanto al montaje de dientes nos interesa solamente destacar el hecho que, cuando se realizan sobredentaduras mandibulares implantomucosoportadas, es aconsejable no montar los segundos molares ya que empeora la estabilidad de la misma. La razón estriba en que, con la reabsorción progresiva que ha ido sufriendo la cresta alveolar, los segundos molares se suelen situar sobre una porción de reborde alveolar que está inclinada y asciende hacia la rama ascendente de la mandíbula. Rara vez el reborde alveolar es aplanado a nivel de los segundos molares inferiores. Desde un punto de vista funcional, este hecho no comporta ningún tipo de problema cuando no hay alimento entre los dientes puesto que también contactan los demás dientes con lo que la estabilidad está garantizada (fig. 20). Ahora bien, cuando el paciente está comiendo y se sitúa alimento entre los segundos molares, sólo existirá contacto en esa zona. Este contacto genera una fuerza que se transmite a un plano inclinado y sabemos que en estas situaciones la fuerza se descompone en otras dos, una dirigida hacia la cresta, que será la responsable de la posterior reabsorción, y otra dirigida hacia mesial, que es la potencialmente desestabilizadora 
(fig. 21). Esta última, produce un desplazamiento en sentido anterior y superior de la sobredentadura, provocando en algunos casos que se clave por lingual de los pilares. Una forma sencilla de comprobar este problema es colocar un rollo de algodón a nivel de los segundos molares y hacer que el paciente ocluya. Lógicamente, esta particularidad del montaje vendrá condicionada por el antagonista ya que si se trata de un diente natural y no forma parte de una prótesis será necesario ofrecerle un contacto oclusal para evitar su extrusión. A modo de alternativa, se ha propuesto montar los segundos molares de manera que el plano oclusal que definan sea paralelo a la cresta, pero no hay que olvidar que la reabsorción progresiva de la misma provocará que se pierda gradualmente ese paralelismo. Por último, cuando se trata de sobredentaduras implantosoportadas se pueden montar los segundos molares inferiores ya que el soporte implantario impide el desplazamiento de la prótesis. En el maxilar superior no es necesario plantearnos esta particularidad en el montaje de dientes puesto que la anatomía de la cresta ósea es más aplanada.

Una vez nos hemos planteado no montar los segundos molares inferiores, debemos analizar hasta que punto nuestra decisión puede afectar a la estética, a la función o a las articulaciones temporomandibulares (ATM).

Desde el punto de vista de la estética, sabemos que cuando un paciente sonríe los principales dientes que son visibles son los dientes superiores $y$, de ellos, en un $7 \%$ se ven solamente los seis dientes anteriores, en un $48,6 \%$ de primer premolar a primer premolar, en un $40,6 \%$ los de segundo premolar a segundo premolar $y$, finalmente, en un 3,7\% de primer molar a primer molar ${ }^{22}$. Por ello, la ausencia de los segundos molares mandibulares no planteará en ningún caso problemas de tipo estético.

Desde el punto de vista de la función, se han realizado múltiples estudios en los que se ha valorado la repercusión tanto en la habilidad como en la capacidad masticatoria en arcadas acortadas (con ausencias de segundos molares o incluso de ambos molares). De forma global, han llegado a la conclusión que la capacidad y habilidad masticatorias no se ven afectadas por el hecho de eliminar los segundos molares, incluso, aunque se eliminen tanto los primeros como los segundos molares ${ }^{23,24}$. De hecho, es sabido que el $90 \%$ de la eficacia masticatoria es generada en la zona de la mitad mesial del primer molar y los dos premolares. Pero, además, resulta que la fuerza que reciben los segundos molares es un $10 \%$ superior a la recibida por los primeros molares. Si tenemos presente que uno de los objetivos de las bases en prótesis implantomucosportada es minimizar la carga que recibe la cresta ósea con el fin de reducir la velocidad de reabsorción de la misma, podemos comprender que, en este sentido, es favorable no montar los segundos molares.

Desde el punto de vista de la repercusión sobre las ATMs, también se ha valorado este aspecto y no se ha hallado relación entre la ausencia de molares y la aparición de patología articular o el empeoramiento de una patología articular preexistente ${ }^{25}$. De hecho, la restauración de los dientes posteriores no conlleva un descenso en la prevalencia de trastornos temporomandibulares ${ }^{25,26}$ y los cambios estructurales a nivel articular serían más bien de tipo adaptativo y no de tipo patológico ${ }^{27.28}$. Además, hay que tener presente que la mayoría de estudios valoran los efectos sobre las ATMs de la pérdida de todos los molares, algo que aquí no se plantea ya que solamente se valora la pérdida de un molar por hemiarcada.

Por tanto, valorando las repercusiones de tipo estético, funcional y articular podemos concluir que es factible realizar montajes eliminando los segundos molares mandibulares sin que ello resulte un perjuicio para el paciente en ninguno de los tres ámbitos mencionados, siempre y cuando el antagonista nos lo permita.

\section{Conclusión}

Cuando se afronta el tratamiento de un paciente mediante una sobredentadura sobre implantes es fundamental tener presentes todos los principios que se siguen cuando se realizan prótesis completas. De hecho, no se puede hacer una buena sobredentadura si no se sabe hacer bien una prótesis completa y no debemos pretender que los implantes subsanen o enmascaren los defectos de nuestra técnica. Junto con esta premisa, será conveniente:

- Tener presente la biomecánica (implantosoportada o implantomucosoportada) ya que condicionará buena parte del diseño de la prótesis (número de implantes, características y tipo de anclaje, fijación del anclaje y montaje de dientes). 
- Elegir el anclaje más sencillo.

- Evitar, en lo posible, los tramos a extensión distal en las barras de sobredentaduras implantomucosoportadas.

- Reforzar siempre las sobredentadu- ras con una estructura de $\mathrm{Cr}$-Co e incluso las prótesis completas antagonistas.

- Fijar los anclajes directamente en la boca cuando la prótesis sea implantomucosoportada.
- No montar los segundos molares inferiores en las sobredentaduras mandibulares implantomucosoportadas, siempre que el antagonista lo permita

\section{Bibliografla recomendada}

Para profundizar en la lectura de este tema, el/los autor/es considera/an interesantes los artículos que aparecen señalados del siguiente modo: *de interés **de especial interés.

1. Deporter D, Watson P, Pharoah M, Todescan R, Tomlinson G. Ten-year results of a prospective study using porous-surfaced dental implants and a mandibular overdenture. Clin Oral Implants Res. 2002;4:1-7.

$2 * *$. Chiapasco M. Early and immediate restorarion and loading of implants in completely edentulous patients. Int $\mathrm{J}$ Oral Maxillofac Implants. 2001;19:76-91.

En este artículo se hace una precisa y realista valoración del estado de la cuestión referente a la carga inmediata, estableciendo las condiciones que se deben dar para llevarla a cabo y marcando las pautas de tratamiento según el tipo de prótesis a realizar.

3. Mericske-Stern R. Force distribution on implants supporting overdentures: the effect of distal bar extensions. A 3-D in vivo study. Clin Oral Implants Res. 1997;8:142-51.

4*. Feine JS, de Grandmont P, Boudrias P, et al. Within-subjects comparisons of implantsupported mandibular prostheses: Choice of prosthesis. J Dent Res. 1994;73:1105-11. Interesante artículo en el que se pone de manifiesto que, a pesar de que con frecuencia pueda parecer la mejor opción de tratamiento una prótesis fija, la facilidad con que se lleva a cabo la higiene debajo de las sobredentaduras las convierte en una modalidad de tratamiento preferible para muchos pacientes.

5. Menicucci G, Lorenzetti M, Pera P, Preti G. Mandibular implant-retained overdenture: Finite element analysis of two anchorage systems. Int J Oral Maxillofac Implants. 1998;3:369-76.

6. Sadowsky SJ, Caputo AA. Effect of anchorage systems and extension base contact on load transfer with mandibular implant-retained overdentures. J Prosthet Dent. 2000;84:32734.

7*. Naert I, Gizani S, Vuylsteke M, Van Steenberghe D. A 5-year prospective randomized clinical trial on the influence of splinted and unsplinted oral implants retaining a mandibular overdenture: Prosthetic aspects and patient satisfaction. J Oral Rehab. 1999;6:195-202. Interesante artículo en el que se valoran, después de 5 años, las complicaciones derivadas del tipo de anclaje utilizado y el grado de satisfacción de los pacientes.
8. Naert I, Gizani S, Vuylsteke M, Van Steenberghe D. A 5-year randomized clinical trial on the influence of splinted and unsplinted oral implants in the mandibular overdenture therapy: Part I. Periimplant outcome. Clin Oral Impl Res. 1998;9:170-7.

9. van Kampen F, Cune M, van der Bilt A, Bosman F. Retention and postinsertion maintenance of bar-clip, ball and magnet attachments in mandibular implant overdenture treatment: an in vivo comparison after 3 months of function. Clin Oral Implants Res. 2003;14:720-6.

10. Stewart BL, Edwards RO. Retention and wear of precision type attachments. J Prosthet Dent. 1983;49:28-34.

11. Jemt T, Lekholm U. Implant treatment of edentulous maxillae: A 5-year follow-up report on patients with different degrees of jaw resorption. Int J Oral Maxillofac Implants. 1995;10:303-11.

12. Hertel RC, Kalk W. Influence of the dimensions of implant superstructure on periimplant bone loss. Int J Prosthodont. 1993; 6:18-24.

13*. Mallat-Desplats E, Mallat-Callís E. Prótesis parcial removible y sobredentaduras. Barcelona: Ed. Elsevier, 2003:373-426.

Se dedica un capítulo entero a sobredentaduras sobre implantes en el cual se describen de forma exhaustiva todos los detalles necesarios para confeccionar sobredentaduras atendiendo a un diseño racional y siempre dependiente de la biomecánica de la prótesis.

14. den Dunnen AC, Slagter AP, de Baat C, Kalk W. Adjustments and complications of mandibular overdentures retained by four implants. A comparison between superstructures with and without cantilever extensions. Int J Prosthodont 1998;11:307-11.

15. Jemt T, Book K, Linden B, Urde G. Failures and complications in 92 consecutively inserted overdentures supported by Branemark implants in severely resorbed edentulous maxillae: a study from prosthetic treatment to first annual check-up. Int J Oral Maxillofac Implants. 1992;7:162-7.

16. Smedberg J, Lothigius E, Bodin I, Frykholm A Nilner K. A clinical and radiological two-year follow-up study of maxillary overdentures on osseointegrated implants. Clin Oral Implants Res. 1993;4:39-46.

17. Langer Y, Langer A. Root-retained overdentures: Part I-Biomechanical and clinical aspects. J Prosthet Dent. 1991;66:784-9.

18. Palmqvist S, Sondele K, Swartz B. Implantsupported maxillary overdentures: Outcome in planned and emergency cases. Int J Oral Maxillofac Implants. 1994;9:184-90.

19. Haraldson T, Jemt T, Stablad PA, Lekholm U. Oral function in subjects with overdentures supported by osseointegrated implants. Scan J Dent Res. 1988;96:235-42.

20. Jacobs R, Van Steenberghe D, Nys M, Naert I. Maxillary bone resorption in patients with mandibular implant-supported overdentures or fixed prostheses. J Prosthet Dent. 1993; 70:135-40.

21. Lechner SK, Mammen A. Combination syndrome in relation to osseointegrated implant supported overdentures: A survey. Int J Prosthodont. 1996;9:58-64.

22. Tjan AHL, Miller GD. Some esthetic factors in a smile. J Prosthet Dent 1984;51:24-8.

23. Carlsson GE. Masticatory efficiency: The effect of age, the loss of teeth and prosthetic rehabilitation. Int Dent J. 1984;34:93-7.

24. Elias AC, Sheiham A. The relationship between satisfaction with mouth and number and position of teeth. J Oral Rehab. 1998; 25:649-61.

25. Witter DJ, de Haan AF, Käyser AF, Van Rossum G. A 6-year follow-up of oral function in shortened dental arches. Part II: Craniomandibular dysfunction and oral comfort. J Oral Rehab. 1994;21:353-66.

26. Pullinger AG, Seligman DA, Gornbein JA. A multiple logistic regression analysis of the risk and relative odds of temporomandibular disorders as a function of common occlusal features. J Dent Res. 1993;72:968-79.

27. Hansson LG, Hansson T, Petersson A. A comparison between clinical and radiologic findings in 259 temporomandibular joint patients. J Prosthet Dent. 1983;50:89-94.

28. Carlsson GE, Droukas BC. Dental occlusion and the health of the of the masticatory system. J Craniomand Pract. 1984;2:142-7. 
RCOE, 2006, Vol 11, N03, 329-343

$$
-344-
$$

